\title{
Local spiral structure based on the Gaia EDR3 parallaxes ${ }^{\star}$
}

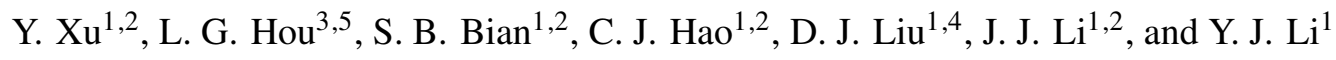 \\ 1 Purple Mountain Observatory, Chinese Academy of Sciences, Nanjing 210023, PR China \\ e-mail: xuye@pmo.ac.cn \\ 2 School of Astronomy and Space Science, University of Science and Technology of China, Hefei 230026, PR China \\ 3 National Astronomical Observatories, Chinese Academy of Sciences, 20A Datun Road, Chaoyang District Beijing 100101, \\ PR China \\ ${ }^{4}$ College of Science, China Three Gorges University, Yichang 443002, PR China \\ 5 CAS Key Laboratory of FAST, National Astronomical Observatories, Chinese Academy of Sciences, Beijing 100101, PR China
}

Received 10 December 2020 / Accepted 30 December 2020

\begin{abstract}
Context. The astrometric satellite Gaia is expected to significantly increase our knowledge as to the properties of the Milky Way. The Gaia Early Data Release 3 (EDR3) provides the most precise parallaxes for many OB stars, which can be used to delineate the Galactic spiral structure.

Aims. We investigate the local spiral structure with the largest sample of spectroscopically confirmed young OB stars available to date, and we compare it with what was traced by the parallax measurements of masers.

Methods. A sample consisting of three different groups of massive young stars, including O-B2 stars, O-B0 stars and O-type stars with parallax accuracies better than $10 \%$ was compiled and used in our analysis.

Results. The local spiral structures in all four Galactic quadrants within $\approx 5 \mathrm{kpc}$ of the Sun are clearly delineated in detail. The revealed Galactic spiral pattern outlines a clear sketch of nearby spiral arms, especially in the third and fourth quadrants where the maser parallax data are still absent. These O-type stars densify and extend the spiral structure constructed by using the Very Long Baseline Interferometry maser data alone. The clumped distribution of O-type stars also indicates that the Galaxy spiral structure is inhomogeneous.
\end{abstract}

Key words. astrometry - Galaxy: structure - stars: early-type - stars: massive - masers

\section{Introduction}

Mapping the Galactic spiral structure has long been a difficult issue in astronomy, because the Sun is deeply embedded in the Galactic plane, resulting in the superposition of multiple structures along the observed line-of-sight. The copious dust extinction makes the situation even worse. In the 1950s, substantial progress in regards to tracing the spiral arm segments in the solar neighborhood was first made by Morgan et al. (1952, 1953) with optically selected high-mass stars (OB stars). Later on, the early $\mathrm{H}$ I surveys in the radio band extended the studies of spiral arms to almost the entire Galactic disk (Christiansen \& Hindman 1952; van de Hulst et al. 1954; Kerr et al. 1957; Westerhout 1957; Oort et al. 1958; Bok 1959). However, it was soon realized that the H I results were not reliable due to the influence of the noncircular motions and the kinematic distance ambiguities. In the 1970s, Georgelin \& Georgelin (1976) proposed a famous model of four-arm segments by using $~ 100$ opticaland radio-selected $\mathrm{H}$ II regions, which was further explored by many researchers by an integrated approach of mass data of various spiral tracers, including high-mass young stars, H II regions, giant molecular clouds, H I clouds, etc. (e.g., Russeil 2003; Hou \& Han 2014). Until now, many approaches have been pur-

\footnotetext{
* Table 1 is only available at the CDS via anonymous ftp to cdsarc.u-strasbg.fr (130.79.128.5) or via http://cdsarc. u-strasbg.fr/viz-bin/cat/J/A+A/645/L8
}

sued to decipher the morphology of the Galaxy spiral arms (e.g., see Xu \& Hou 2018a; Shen \& Zheng 2020, for reviews). There is a general consensus that a global spiral pattern exists in the Galactic disk between Galactocentric distance of about 3 and $10 \mathrm{kpc}$. However, considerable disagreements remain in regards to the finer details. The arm geometries, orientations, and even the number of arms are still debated.

Measuring the distances as accurately as possible for many spiral tracers would be the key to settle the disputes. Through measuring the trigonometric parallax of masers in high-mass star formation regions, the Very Long Baseline Interferometry (VLBI) has achieved a revolutionary breakthrough in the spiral structure of the Milky Way (Xu et al. 2006). The VLBI can now obtain parallax accuracies down to a few $\mu$ as (e.g., Sanna et al. 2017). Accurate trigonometric parallaxes for about 200 high-mass star formation regions have been measured. They are spread over about one-third of the entire Galactic disk, which is the subject of an updated view of the Galactic spiral structure that has recently been proposed by Reid et al. (2019). However, there is still a lack of observational data for many Galaxy areas, especially in the fourth Galactic quadrant. In order to accurately trace the extension of nearby spiral arm segments, the parallax measurements of masers need to be supplemented.

By taking advantage of the second data release of the Gaia mission, (Xu et al. 2018b, hereafter Paper I) extended the nearby spiral arms from the first and second Galactic quadrants to the 
Table 1. Parallaxes and proper motions of OB stars from Gaia EDR3.

\begin{tabular}{lccccccc}
\hline \hline Name & Gaia EDR3 ID & $\begin{array}{c}\text { RA } \\
\left({ }^{\circ}\right)\end{array}$ & $\begin{array}{c}\text { Dec } \\
\left({ }^{\circ}\right)\end{array}$ & $\begin{array}{c}\pi \\
(\mathrm{mas})\end{array}$ & $\begin{array}{c}\mu_{x} \\
\left(\mathrm{mas} \mathrm{yr}^{-1}\right) \\
(6)\end{array}$ & $\begin{array}{c}\mu_{y} \\
\left(\mathrm{mas} \mathrm{yr}^{-1}\right) \\
(7)\end{array}$ & $\begin{array}{c}\text { Spectral } \\
\text { type } \\
(8)\end{array}$ \\
\hline LS I +66 3 & 528563384392653312 & 0.1219 & 67.2168 & $0.948 \pm 0.012$ & $-1.80 \pm 0.01$ & $-2.43 \pm 0.01$ & OB \\
[B55b] 5542 & 429950454253663360 & 0.1471 & 62.5549 & $0.413 \pm 0.023$ & $-3.55 \pm 0.02$ & $-1.17 \pm 0.03$ & B2 \\
[B53] 368 & 423149081478526464 & 0.2867 & 58.9799 & $0.261 \pm 0.015$ & $-3.32 \pm 0.01$ & $-2.70 \pm 0.02$ & B0 \\
LS I +64 9 & 431771623466692608 & 0.3067 & 64.5883 & $0.332 \pm 0.015$ & $-2.76 \pm 0.01$ & $-0.83 \pm 0.01$ & B2V \\
[B53] 369 & 423157774492279680 & 0.3113 & 59.1428 & $0.264 \pm 0.014$ & $-3.02 \pm 0.01$ & $-1.50 \pm 0.01$ & B2
\end{tabular}

Notes. Column (1) is the star name given in Skiff (2014); Col. (2) lists the Gaia EDR3 ID of the matched source; Cols. (3) and (4) are the right ascension (RA) and declination (Dec) given by Gaia EDR3, and the reference epoch is J2016.0; Col. (5) is the Gaia parallax and its $1 \sigma$ uncertainty; Cols. (6) and (7) list the proper motions in the eastward $\left(\mu_{x}=\mu_{\alpha} \cos \delta\right)$ and northward $\left(\mu_{y}=\mu_{\delta}\right)$ directions, respectively, also given are their $1 \sigma$ uncertainties; Col. (8) is the spectral type derived from Skiff (2014). The full table is available at the CDS.

third and fourth quadrants. The spiral structure within $\approx 3 \mathrm{kpc}$ of the Sun was well depicted with massive, young stars (OB stars). To accurately extend the nearby arm segments to more distant regions ( $~ 23 \mathrm{kpc}$ ), higher accuracies of parallax data are crucial. Recently, the Gaia mission released part of the third data set (the Gaia Early Data Release 3, hereafter Gaia EDR3). The astrometric data (Gaia Collaboration 2016, 2021) have been updated significantly, up to a parallax accuracy of 20-30 $\mu$ as, which enables us to reveal the detailed local spiral structure in a wider range $(\approx 5 \mathrm{kpc})$.

\section{OB star sample}

The OB stars are taken from the catalog of stellar spectral classifications of Skiff (2014), which has been updated up to 2020 February 6. This catalog represents 68612 spectroscopically confirmed OB stars collected from the literature, along with spectral type classifications and accurate coordinates $\left(\approx 1^{\prime \prime}\right)$. It is probably the largest sample of spectroscopically confirmed OB stars available to date, which enables us to reveal the spiral structure within $\approx 5 \mathrm{kpc}$ of the Sun. However, this catalog is not complete, more OBs have yet to be identified from Gaia or other photometric and spectroscopic surveys. We carried out a crossmatch between these OB stars and the Gaia EDR3 catalog with a match radius of $1^{\prime \prime}$. After eliminating the targets that had more than one positionally matched Gaia source, we found 14414 $\mathrm{O}-\mathrm{B} 2$ stars.

We noticed that many of the 14414 O-B2 stars have considerable parallax uncertainties, that is to say larger than $10 \%$, which are comparable with the typical spacing between the nearby spiral arm segments. Therefore, similar to Paper I, we only adopted the O-B2 stars with distance accuracies better than $10 \%$ to depict the nearby spiral arms. After rejecting stars with vertical heights larger than $300 \mathrm{pc}$, we obtained a reduced subsample of 9750 sources that includes 5386 O-B0 stars and 1089 O-type stars. Their Gaia source ID, coordinates, parallaxes, proper motions, and also the names and spectral type classifications from Skiff (2014) are listed in Table $1^{1}$. In comparison, there were only $2800 \mathrm{OB}$ stars with parallax accuracies better than $10 \%$ in Paper I. For the OB stars in Table 1, their Gaia $G$ band apparent magnitudes are between 2.6 and 18.3, $\sim 87 \%$ of them have $G$ magnitudes $<12$. They are distributed in

\footnotetext{
1 https://cdsarc.u-strasbg.fr/ftp/vizier.submit/ /xygaia2020v2/
}

the Galactic longitude range from $0^{\circ}$ to $360^{\circ}$, about $90 \%$ of them are located in Galactic latitude $|b|<5^{\circ}$.

There is an average systematic bias of 0.017 mas on the parallax zero-point of Gaia EDR3. How to properly correct a subsample of Gaia stars for this bias is not a closed issue. We tested its impact on our results by correcting the average systematic bias to the stars, and we found that the influence is very small for the nearby OB stars studied in this work. In the following analysis, we neglect the bias on the Gaia parallax zero-point for the OB stars.

\section{Spiral structure}

In this section, we investigate the nearby spiral structure traced by three different groups of OB stars, that is, the O-B2 stars, the O-B0 stars, and only the O-type stars. As shown in the left panel of Fig. 1, the distribution of $\mathrm{O}-\mathrm{B} 2$ stars projected onto the Galactic plane clearly delineate three spiral arm segments, they are the Perseus Arm, the Local Arm, and the SagittariusCarina Arm from top to bottom. In Paper I with Gaia DR2 data, the scopes of these arm segments were only limited to within $\approx 3 \mathrm{kpc}$ of the Sun. While with the recently released Gaia EDR3 data, these arm segments extend to more distant Galaxy regions, $\approx 5 \mathrm{kpc}$ away from the Sun as shown in Fig. 1. Especially in the fourth Galactic quadrant, the Sagittarius-Carina Arm was traced to $(X \sim-7.0, Y \sim 5.5) \mathrm{kpc}$ by O-B2 stars, as far as $7.5 \mathrm{kpc}$ from us. In the spiral arms, we notice that the distribution of $\mathrm{O}-\mathrm{B} 2$ stars is uneven, which may be a result of the uneven distribution of giant molecular clouds (e.g., Dame et al. 2001; Sun et al. 2019) and/or foreground extinction. Meanwhile, besides the major spiral arm segments, there are quite a lot of stars scattered in the inter-arm regions.

A peculiar motion speed of a star is normally from a few to tens of $\mathrm{km} \mathrm{s}^{-1}$. The B2-type stars can live for $\sim 20$ million years, which means that they could migrate far from their birthplaces, up to about $1 \mathrm{kpc}$. If their peculiar motions are perpendicular to spiral arms, a rather large portion of the B2-type stars born in spiral arms would already have migrated far from the birth sites. In comparison, the lifetimes of B0-type stars are less than about ten million years, only some of them could leave the spiral arms where they were born. As shown in the right panel of Fig. 1, relatively few stars are located in the inter arm regions while we made a similar plot with O-B0 stars, rather than O-B2 stars.

The more massive a star is, the younger it is, and the better tracer it is for the Galaxy spiral arms. Because of their shorter life spans than the B0-B2 stars, generally speaking, most of the 

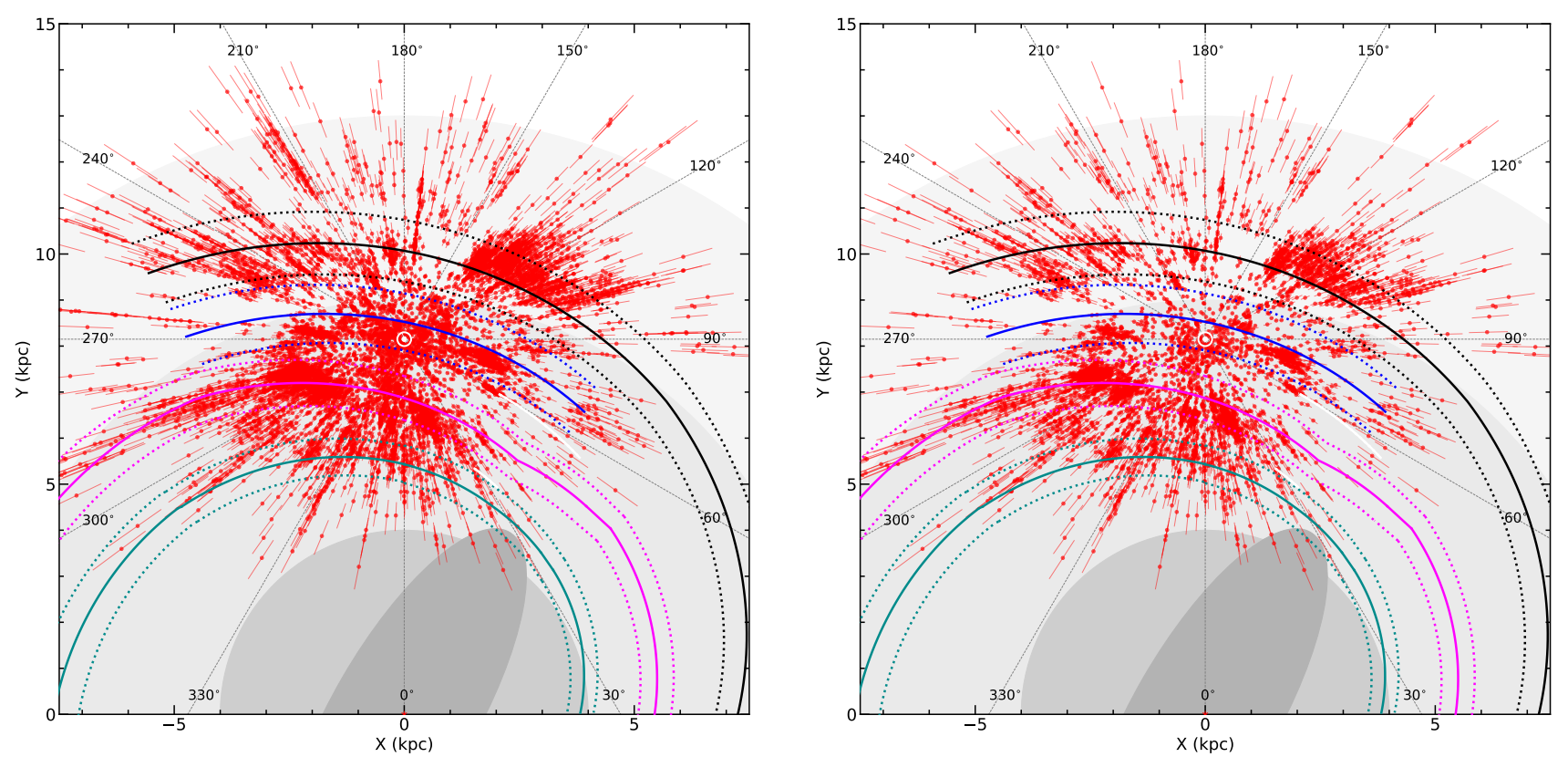

Fig. 1. Locations of the O-B2 stars (red dots, left) and O-B0 stars (red dots, right) projected onto the Galactic plane, together with their $1 \sigma$ distance uncertainties. The Sun (Sun symbol) is at $(0,8.15) \mathrm{kpc}$. Only the stars with formal parallax uncertainties better than $10 \%$ are shown. The solid and dashed curved lines denote the arm center and widths (enclose 90\% of the masers) fitted by Reid et al. (2019) from their parallax data of VLBI masers. The Perseus Arm (black), the Local Arm (blue), the Sagittarius-Carina Arm (magenta), the Scutum-Centaurus Arm (dark cyan), and two spur-like structures (white) are indicated with different colors. Straight dashed lines (gray) denote the Galactic longitudes.

O-type stars are still located near their birthplaces and, consequently, can be a better tracer for Galaxy spiral structure. In Fig. 2, we solely focus on the distribution of O-type stars. In order to depict the entire pattern of spiral arms, the high-mass star formation region masers with VLBI parallax measurements (Reid et al. 2019) are also displayed, which distribute in a much broader region than that of O-type stars in the first Galactic quadrant. By combining these high-quality data, the properties of nearby spiral arms are explored and discussed below.

Along the Perseus Arm, the O-type stars tend to cluster. Many O-type stars accumulate between Galactic longitude $l \sim$ $210^{\circ}$ and $l \sim 250^{\circ}$, despite it being thought that a gap was $\bar{c}$ there. Together with clumps of O-type stars around $l \sim 180^{\circ}$ and clumps located between $l \sim 100^{\circ}$ and $l \sim 150^{\circ}$, these results indicate the uneven star formation in the Perseus Arm. Interestingly, in the third quadrant, the Perseus Arm traced by O-type stars tends to spiral inward toward the Galactic center direction in comparison to that defined by a small number of known VLBI masers. In addition to pointing away and being traceable between heliocentric distances of 0.3 and $1.5 \mathrm{kpc}$, the Orion Spur protrudes from this major feature between $l \sim 210^{\circ}$ and $l \sim 220^{\circ}$.

Remarkably, the Local Arm traced by the distribution of O-type stars is distinct, it extends much longer than previously expected, and it seems more similar to a major spiral arm feature. It extends to the third and fourth Galactic quadrants almost directly at $l \sim 260 / 270^{\circ}$, with a length of $\sim 3 \mathrm{kpc}$ from the Sun, and perhaps it would extend further and spiral inward to the fourth quadrant. In addition, there is an obvious difference between the distribution of O-type stars and the modeled arm extension by using VLBI masers. The Local Arm traced by O-type stars seems to present a downward drift toward the Galactic center in comparison of the maser measurements. In the longitude range of $l \sim 240^{\circ}-330^{\circ}$, there is still a lack of VLBI maser data, the upcoming BeSSeL Survey to the southern hemisphere would solve the mystery (Reid et al. 2019).

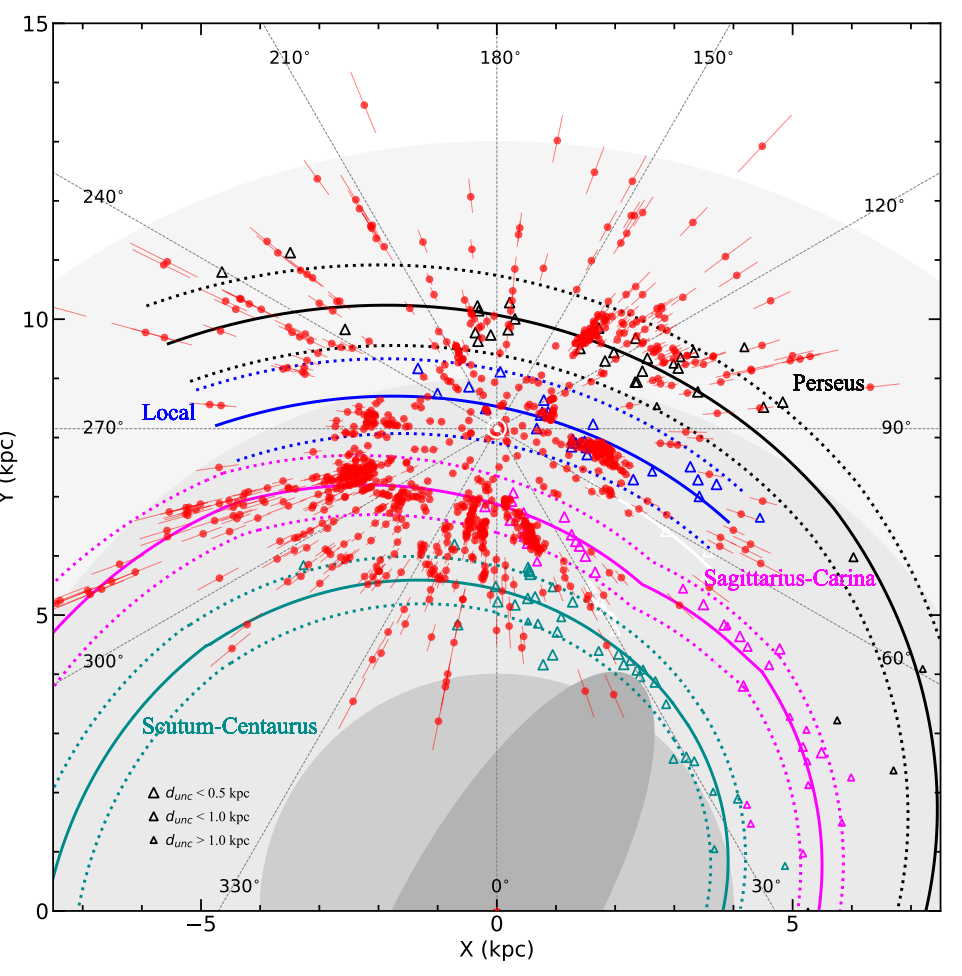

Fig. 2. Locations of the O-type stars in Gaia EDR3 (red dots) and the masers (triangles) from Reid et al. (2019). The formal parallax uncertainties of the O-type stars shown here are better than $10 \%$. See the caption of Fig. 1 for more details.

The Sagittarius-Carina Arm delineated by the distribution of O-type stars is shown as an arc-shaped structure: from $l \approx 50^{\circ}$ through $l=0^{\circ}$ to $l \approx-80^{\circ}$, between $R \sim 6 \mathrm{kpc}$ and $\sim 9 \mathrm{kpc}$ with a length of $\approx 10 \mathrm{kpc}$, where $R$ is the Galactocentric radius. 


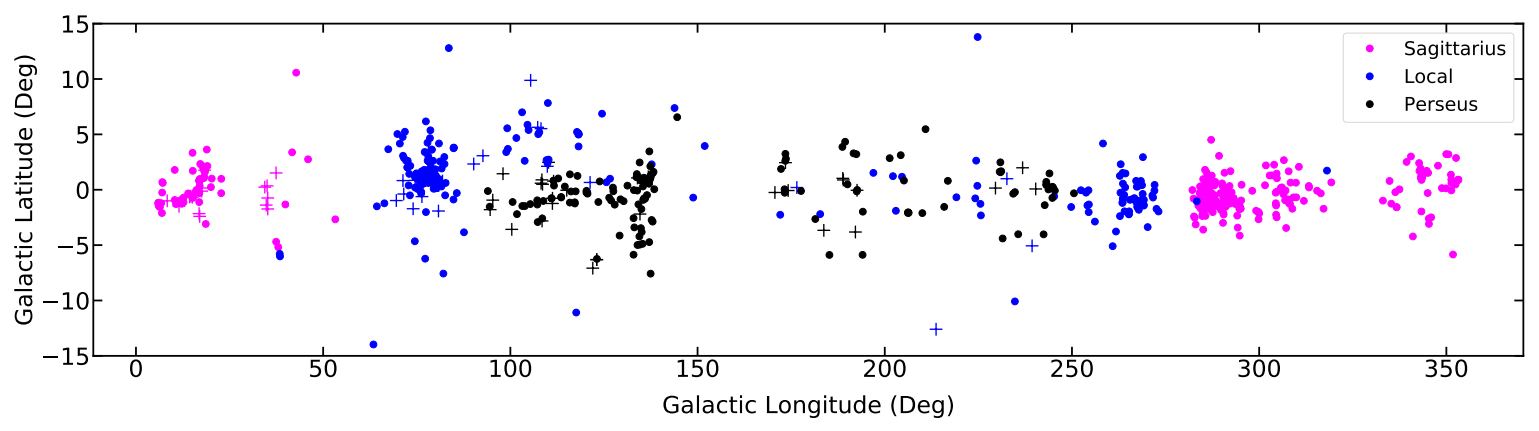

Fig. 3. Galactic distribution of the O-type stars from Gaia EDR3 (dots) and the VLBI masers (crosses, Reid et al. 2019). Only the O-type stars with parallax uncertainties $<10 \%$ are shown. Sources located in the Perseus Arm (black), the Local Arm (blue), and the Sagittarius-Carina Arm (magenta) are indicated by different colors.

Strikingly, in the fourth quadrant, the distribution of O-type stars in the Sagittarius-Carina Arm is well consistent with the model prediction of arm extension given by the VLBI masers (Reid et al. 2019). Meanwhile, the Scutum-Centaurus Arm is also discernable from $l \approx 25^{\circ}$ through $l=0^{\circ}$ to $l \approx-50^{\circ}$ in the distribution of O-type stars.

In short, the O-type stars tend to clump together and some of them are frequently found between the major spiral arms, indicating that recent star formation does occur in the inter arm regions and/or some of them have migrated far away from their birthplaces. The nearby spiral pattern, on the whole, is similar from both VLBI and Gaia parallax measurements in the range of $0^{\circ}<l<240^{\circ}$. Meanwhile, as shown in Fig. 3, the distributions of O-type stars in the Galactic latitude almost resemble the VLBI masers, indicating that the O-type stars indeed trace the spiral arms similar to the masers. In Fig. 3, to assign the O-type stars to the nearby spiral arms, we adopted the best-fitted arm center and arm widths (enclose $90 \%$ of the VLBI maser sources) from Reid et al. (2019).

\section{Conclusions}

The VLBI parallax measurements of masers have nicely traced the Galactic spiral structure in the first, second, and third Galactic quadrants. While there is still a lack of maser data in about two-thirds of the entire Galactic disk, especially for the fourth quadrant. The OB stars with accurate Gaia parallaxes can densify and extend the current picture, in particular in the Galaxy area within $\approx 5 \mathrm{kpc}$ of the Sun. In this work, with the compiled O-type stars, the Perseus Arm, the Local Arm, the SagittariusCarina Arm, and the Scutum-Centaurus Arm are extended to the third or even fourth Galactic quadrants with high confidence, where the maser data are still rare. The O-type stars tend to clump together, largely as a result of the state of their ancestors, the giant molecular clouds, indicating that the Milky Way presents a discrete or uneven spiral structure. The ubiquitousness of spurs traced by O-type stars also indicate that our Galaxy may not have a pure grand design morphology.

Acknowledgements. This work was sponsored by the MOST under grant No. 2017YFA0402701. This work was funded by the NSFC Grands 11933011, 11873019 and 11673066, 11988101 and the Key Laboratory for Radio Astronomy. L.G.H. thanks the support from the Youth Innovation Promotion Association CAS. This work has made use of data from the European Space Agency (ESA) mission Gaia (https://www.cosmos.esa.int/gaia), processed by the Gaia Data Processing and Analysis Consortium (DPAC, https://www . cosmos.esa.int/web/gaia/dpac/consortium). Funding for the DPAC has been provided by national institutions, in particular the institutions participating in the Gaia Multilateral Agreement.

\section{References}

Bok, B. J. 1959, The Observatory, 79, 58

Christiansen, W. N., \& Hindman, J. V. 1952, Aust. J. Sci. Res. A Phys. Sci., 5, 437

Dame, T. M., Hartmann, D., \& Thaddeus, P. 2001, ApJ, 547, 792

Gaia Collaboration (Prusti, T., et al.) 2016, A\&A, 595, A1

Gaia Collaboration (Brown, A. G. A., et al.) 2021, A\&A, in press, https:// doi .org/10.1051/0004-6361/202039657

Georgelin, Y. M., \& Georgelin, Y. P. 1976, A\&A, 49, 57

Hou, L. G., \& Han, J. L. 2014, A\&A, 569, A125

Kerr, F. J., Hindman, J. V., \& Carpenter, M. S. 1957, Nature, 180, 677

Morgan, W. W., Sharpless, S., \& Osterbrock, D. 1952, AJ, 57, 3

Morgan, W. W., Whitford, A. E., \& Code, A. D. 1953, ApJ, 118, 318

Oort, J. H., Kerr, F. J., \& Westerhout, G. 1958, MNRAS, 118, 379

Reid, M. J., Menten, K. M., Brunthaler, A., et al. 2019, ApJ, 885, 131

Russeil, D. 2003, A\&A, 397, 133

Sanna, A., Reid, M. J., Dame, T. M., Menten, K. M., \& Brunthaler, A. 2017, Science, 358, 227

Shen, J., \& Zheng, X. W. 2020, Res. Astron. Astrophys., 20, 159

Skiff, B. A. 2014, VizieR Online Data Catalog: B/mk

Sun, Y., Yang, J., Xu, Y., et al. 2019, ApJS, 246, 7

van de Hulst, H. C., Muller, C. A., \& Oort, J. H. 1954, Bull. Astron. Inst. Neth., 12,117

Westerhout, G. 1957, Bull. Astron. Inst. Neth., 13, 201

Xu, Y., Reid, M. J., Zheng, X. W., \& Menten, K. M. 2006, Science, 311, 54

Xu, Y., Hou, L. G., \& Wu, Y. W. 2018a, Res. Astron. Astrophys., 18, 146

Xu, Y., Bian, S. B., Reid, M. J., et al. 2018b, A\&A, 616, L15 